Disease diagnosis and management

\section{European evidence-based consensus on the diagnosis and management of Crohn's disease}

\section{S B Hanaver, W J Sandborn}

\section{When is a consensus not a consensus?}

$\mathrm{E}$ duard Stange and Simon Travis ${ }^{1}$ are to be congratulated on their magnum opus encompassing 234 statements and 727 references pertaining to the diagnosis and management of Crohn's disease. The aim of the consensus was not to "supersede guidelines of different countries", but to "promote a European perspective on the management of Crohn's disease" under the auspices of the European Crohn's and Colitis Organization (ECCO). ECCO is a forum established in 2000 for specialists interested in inflammatory bowel disease (IBD) to promote European views, clinical trials and specialist training in IBD. We agree with many, if not most, of the statements and recommendations, and note that the European consensus with regard to diagnosis and management of Crohn's disease is substantially aligned with practice guidelines from the US. ${ }^{2}$

The methods (strategy) of the Consensus entailed five steps. Fourteen separate topics were assigned to 13 working parties whose chairmen developed questions that were distributed to the working parties. The working parties dealt with the questions on the basis of their experience and evidence from the literature (gained from a systematic literature search). An evidence level was graded (1-5) according to the guidelines of the Oxford Centre for Evidence Based Medicine (Oxford, UK). Provisional guideline statements were then written by the chairmen and circulated among the working parties, which met, first, in Prague at the 2004 United European Gastroenterology Week Conference, where 61 participants from 20 countries gathered to agree on the final versions of each guideline statement. The participants continued to discuss the individual statements until a consensus $(>80 \%$ agreement) was reached. Each statement was then given a recommendation grade (A-D) on the basis of the level of evidence, and a final document for each topic was written by the chairmen in conjunction with their working party. The final text was edited by Drs Stange and Travis.
The document is organised into 14 main headings ranging from diagnosis, through medical and surgical management within the spectrum of common disease scenario, and concluding with special situations such as postoperative treatments, management of fistulae and extraintestinal manifestations, and a potpourri of topics such as management of disease in pregnancy, management of paediatric patients, psychosomatics and alternative treatment. Needless to state, the available literature and level of evidence vary among topics. By necessity, the recommendation grades also vary widely between topics. Furthermore, with the number of chairs and working parties, the consistency of assignment of evidence and recommendation grade also varies within and between statements.

Within the section on "definitions and diagnosis", there were 37 statements. The breakdown according to levels of evidence was $1(16 \%), 2(35 \%), 3(2 \%), 4(0)$ and 5 $(46 \%)$; and recommendation grades was A (16\%), B (35\%), C (2\%) and D (17\%).

Within the section on "current management", there were 64 statements with levels of evidence 1 (44\%), 2 (17\%), 3 $(6 \%), 4(17 \%)$ and $5(16 \%)$, and recommendation grades of A $(20 \%)$, B $(30 \%)$, C (19\%) and D (31\%).

Within the section on "special situations", there were 133 statements with levels of evidence 1 (16\%); 2 (29\%), 3 (16\%), $4(22 \%)$ and 5 (17\%); and recommendation grades of A $(6 \%), \mathrm{B}(22 \%), \mathrm{C}$ $(28 \%)$ and D (34\%).

Thus, the consistency between assigned levels of evidence and translation to recommendations also varied within working parties. In addition, despite the attempt to minimise the overlap between working parties, there were topics where differences in levels of evidence (and their interpretations) led to inconsistencies regarding recommendation grades.

After an introduction regarding methods (including tables on the Oxford Centre's Levels of Evidence and Grades of Recommendation), the Consensus
Document begins with definitions of disease activity according to the Crohn's Disease Activity Index (CDAI). We would disagree with the Consensus Document with respect to the requirement that clinical response should be defined as a decrease in the CDAI $\geqslant 100$ points. The 100-point change tracks more closely to remission $(\mathrm{CDAI}<150)$ points than it does to an alternative response definition of a decrease in the CDAI $\geqslant 70$ points, a definition that may be more sensitive to clinical benefit. Another weakness of the Consensus Document is that there is minimal reference to how the CDAI applies to clinical presentation or interpretation. We have found that working clinical definitions of remission, mild, moderate and severe disease have been very useful with respect to applying the results of clinical trials to clinical practice. ${ }^{2}$ Finally, in contrast with North American guidelines, steroid dependence is defined as the inability to taper the equivalent of prednisolone (or budesonide) $<10 \mathrm{mg} /$ day (or $3 \mathrm{mg} /$ day), compared with complete taper. In the modern era, where there are multiple steroid-sparing options available, we would suggest that steroid dependence be defined as the inability to completely discontinue prednisone (or equivalent). We would also make the point that although prednisone dependence is not acceptable, maintenance treatment with budesonide (budesonide dependence) may be accepted because of the relatively more favourable safety profile.

The ECCO statements begin by describing the clinical features, diagnosis, history and laboratory features of Crohn's disease. Clinicians, investigators and regulatory authorities should not feel "bound" by the statements and recommendations in this section of the Consensus Document, as these are clearly expert opinions based on level 5 evidence. Confirmation of the diagnosis and extent of Crohn's disease is best obtained by ileocolonoscopy (ELl, RGA). Radiological studies are necessary when endoscopic intubation of the intestine is not possible (ELl), and complementary imaging via ultrasound, computed tomography and magnetic resonance imaging are recommended to investigate extramural complications (ELl). There is less evidence to assess the number of endoscopic biopsies necessary to establish a diagnosis or the handling and interpretation of biopsies. Individual histological features (architecture, chronic inflammation, polymorph inflammation, crypt and epithelial changes, etc) are reviewed without assessment of levels of evidence, similar to the statement that "microscopic features for the diagnosis of dysplasia... are the same as those proposed for ulcerative colitis" and despite the statement that 
"these studies on ulcerative colitis have not been replicated in Crohn's disease". Further statements regarding surgical pathological assessment are clearly recommendations from expert pathologists without supporting evidence.

The authors recognise the absence of evidence-based recommendations regarding the utilities of genetic, serologic or phenotype-driven classifications, but do propose evaluation of $\mathrm{C}$ reactive protein to identify patients at higher risk of relapse (EL2) and discuss other, less disseminated assays (such as orosomucoid, IL-6, permeability, calprotectin, lactoferrin, etc) that are currently being evaluated as markers of disease activity.

Not surprisingly, the consensus on current management contains more evidence from therapeutic clinical trials. Here the evidence clearly favours budesonide for mild-moderate active ileocaecal disease (more effective than placebo and mesalazine and less side effects than prednisolone; EL1) and sulfasalazine for mild-moderate active colonic disease (more effective than placebo and less side effects than prednisolone; ELl). Use of oral mesalamine and antibiotics (apart from treatment of septic complications), although commonly used in practice in the US, is not supported by evidence (EL5). Severely active localised (or extensive) disease is treated with systemic corticosteroids or infliximab (ELl). These recommendations differ from the most recent practice guidelines from the US published in 2001 with respect to mesalamine, budesonide and antibiotics. ${ }^{2}$ These differences represent partly assimilation of newer data from clinical trials with oral mesalamine and in part application of evidence-based medicine to clinical practice. $^{4}$

In the section on medical management maintenance therapy (6.2.1) mesalazine is not recommended for maintenance of medically induced remission (ELl), which again would differ from the most recent practice guidelines from the US published in 2001.2 Although we agree that this recommendation represents the best available evidence, we see "human nature" at work in Section 6.3.2 "after a first presentation if remission is achieved medically, maintenance with mesalazine is a treatment option, although there is no consistent evidence for efficacy" (ELl, RGD). The low recommendation grade in view of "no evidence for efficacy" likely indicates advocation of oral mesalazine in this setting based on their clinical experience and safety and the lack of satisfactory therapeutic alternatives. The maintenance statements also advocate the use of azathioprine/mercaptopurine or methotrexate "for those who have relapsed (after steroids)". This exempli- fies one problem with the structure of the consensus document-that is, it fails to view Crohn's disease as a chronic disease requiring both inductive and maintenance treatment. Clearly, approximately $80 \%$ of patients in maintenance trials with steroids, azathioprine or infliximab develop a recurrence within 12 months of terminating acute treatment. Hence, the decision to embark on budesonide, corticosteroids, azathioprine or infliximab requires a plan for long-term, rather than short-term treatment. The concept of induction-maintenance sequence is discussed with respect to the recent debates regarding "top-down" versus "step-up" approaches, but is not consistently applied through the document.

Although the document assigns steroid treatment for extensive small bowel disease an evidence level of (ELl), there are no supporting trials or long-term strategies that have been prospectively assessed for treatment of jejunoileitis (similar to treatment of oesophageal and gastro-duodenal disease). Treatment of steroid-refractory disease does have supporting data for azathioprine/mercaptopurine and methotrexate, although, according to the consensus definition of steroid-refractory disease, there have been no trials supporting azathioprine in this setting. As azathioprine requires months to work, until there is evidence of benefit in steroidrefractory disease, either methotrexate or infliximab would be stronger options.

The section on therapy-specific considerations is extensive and discusses most of the evidence for each specific class of agents (aminosalicylates, antibiotics, corticosteroids, infliximab, thiopurines, methotrexate and investigational agents). There are a few inconsistencies. For instance, although the document rejects antibiotics for treatment of Crohn's disease, data on ciprofloxacin are presented without comment as having "similar efficacy to mesalazine" and "ciprofloxacin therapy may be extended for 6 months (or) until side effects occur". The section on monitoring of corticosteroid treatment includes osteoprotective treatment, but only supplements of vitamin D and calcium are discussed (in this section), whereas in reality there are no data relating to these effects on steroid-induced osteopenia and conversely no data are provided (in this section) regarding the use of bisphosphonates for this treatment indication.

The section on management of medically induced remissions is formatted in a manner opposite to that on medical management of active disease by specifying data for each individual agent before establishing general principles. The discussion on infliximab propones azathioprine to reduce immunogenicity but considers episodic "on demand" treatment to be an effective strategy, without discussion of the risks of increased immunogenicity and gradual loss of response with the episodic strategy. We believe that the recommendation that episodic treatment with infliximab is an acceptable treatment strategy represents European opinion (possibly related to toxicity, economic and regulatory issues) and not the best available evidence. Another controversial statement pertains to potential long-term treatment with mesalazine to "reduce the risk of colon cancer", which is contrary to the conclusion regarding mesalazine as a therapeutic agent and is based on level 4 evidence in ulcerative colitis.

The section on surgery is more descriptive, most likely related to less available evidence and the absence of a surgery cohort in the consensus working groups. A recommendation that "corticosteroids should be weaned if possible" before surgery due to the increased risk factor for surgical complications is not practical as steroid dependency is considered an indication for surgery.

The final section on special situations is elaborate. It reviews data regarding postoperative recurrence and advocates mesalazine as "The drug of choice (ELl, RGB)" although the review of data states that "available data do not demonstrate a robust effect for any medical therapy". The question arises as to how prophylaxis against recurrence with mesalazine is assigned level 1 evidence in view of the conclusion of no robust effect. Further confusion derives from the concluding statement on mesalazine that "given mesalazine's limited effect, no prophylactic treatment may be an option in some asymptomatic...patients". Again, we see "human nature" at work; although the authors know that evidence is lacking, they nevertheless do advocate the use of oral mesalazine as postoperative maintenance treatment, presumably based on their clinical experience and their commitment to the historical data regarding aminosalicylates. Furthermore, the concept of prophylaxis applies only to patients who are asymptomatic; otherwise it should be considered treatment of active disease.

Further special situations that are extensively reviewed are fistulating disease, disease in children and adolescents, and management of pregnancy. The statements are as "evidence-based" as possible in these less formally evaluated scenarios. The consensus document also covers psychosomatic issues and extraintestinal complications and provides a thorough review of the epidemiology and evidence related to treatment, as it exists. Here the discussion regarding osteoporosis is much more comprehensive than the statement pertaining to corticosteroid monitoring. 
Finally, the document concludes with a discussion of alternative treatments that are so commonly used by patients.

The ECCO evidence-based consensus is an outstanding effort to assimilate a multinational European viewpoint regarding the diagnosis and treatment of Crohn's disease and for the most part the effort succeeded admirably. Although the application of evidence levels and recommendation grades is somewhat uneven, the evidence is provided in an unbiased manner to allow readers to establish their own basis for decision making. Furthermore, the large numbers of statements assigned evidence levels of 4-5 are ripe for future, hypothesisgenerating clinical studies.

The diagnosis and treatment of Crohn's disease is on the verge of undergoing evolutionary changes with respect to advances in genetics, serology, understanding of the innate and adaptive immune responses and the resultant molecular targets for therapeutics. In addition, the disparate therapeutic approaches to ulcerative colitis and Crohn's disease are undergoing a convergence from similar advances in aetiopathogenesis and treatment-for example, the expanding role of tumour necrosis factor treatment. The future treatments for these diseases will, eventually, be determined by genetic, serologic and proteomic standards that are yet to be determined. Ultimately, such techniques should define optimal inductive and maintenance strategies, possibly based on individualised profiles. In the mean time, assimilation of our current evidence and application of international standards, as complied by the ECCO evidence-based consensus document, highlights both the convergence of evidence and the areas in need of higherquality data.

Gut 2007;56:161-163.

doi: 10.1136/gut.2005.089953

\section{Authors' affiliations}

S B Hanauer, W J Sandborn, University of Chicago Pritzker School of Medicine, Chicago, Illinois, USA

Correspondence to: Professor S B Hanaver, University of Chicago, MC 4076, 5841 S.
Maryland Ave, Chicago, IL 6061 1, USA; shanaver@uchicago.edu

Competing interests: SBH, Procter and GambleResearch support, consultant, CME activities. Shire-Research support, consultant, CME activities. Prometheus Laboratories-Research support, consultant, CME activities. CentocorResearch support, consultant, CME activities. Schering-Plough-CME activities. Ferring-CME activities. WJS, Procter and Gamble-Research support, consultant, CME activities. ShireResearch support, consultant, CME activities. Prometheus Laboratories-Consultant, CME activities. Centocor-Research support, consultant, CME activities.

\section{REFERENCES}

1 Stange EF, Trauis SPL, Vermeire S, et al. European evidence based consensus on the diagnosis and management of Crohn's disease: definitions and diagnosis. Gut 2006;55(Suppl I):i1-15.

2 Hanaver SB, Sandborn W. Management of Crohn's disease in adults. Am J Gastroenterol $2001 ; 96: 635-43$.

3 Sandborn WJ, Fazio WW, Feagan BG, et al. AGA technical review on perianal Crohn's disease. Gastroenterology 2003;125:1508-30

4 Sandborn WJ, Feagan BG. Review article: mild to moderate Crohn's disease--defining the basis for a new treatment algorithm. Aliment Pharmacol Ther 2003; 18:263-77.

\section{EDITOR'S QUIZ: GI SNAPSHOT}

\section{Acute abdomen after prolonged plane travel}

\section{Clinical presentation}

A 69-year-old woman developed an acute abdomen immediately after a prolonged ( $>12-\mathrm{h})$ flight. At laparotomy, two abnormal segments of jejunum were found: one $16 \mathrm{~cm}$ long, immediately distal to the ligament of Treitz; the other $4 \mathrm{~cm}$ long, $20 \mathrm{~cm}$ distal to the first. Both were plum-coloured, swollen and sharply demarcated from the adjacent normal bowel. There was mild acute peritonitis. The jejunal arterial supply was intact. There was no volvulus or adhesion. Both segments were resected, anastomoses were performed, and the patient made an uneventful recovery.

The patient's history included aortic valve replacement, coronary artery bypass, cholecystectomy and 30 years of smoking. She had taken warfarin sodium since her valve replacement 9 years earlier, and had occasional episodes of epistaxis. At presentation, her international normalised ratio was 5.5 (preferred level, 2-3). Other drugs included carvedilol, valsartan and indapamide. There was no history of abdominal trauma.

\section{Question}

How can these pathological findings, which were present in both segments, be explained? See page 242 for answer

M Yee, H Ye, A B West, Department of Pathology, New York University, New York, New York, USA

Correspondence to: Dr A B West, AmeriPath New York Gastrointestinal Diagnostics, 1 Greenwich Place, Shelton, CT 06484, USA; brian.wes@@med.nyu.edu 